\title{
An Unusual Peripherally Inserted Central Catheter (PICC) Fractured in Vivo with Embolization Happened in a Child: A Case Report
}

\author{
Fang Fang, Hongyan Zhang, Wu Yang \\ Department of Hematology, The Affiliated Hospital of Qingdao University, Qingdao, China \\ Email: fasjp@163.com
}

Received 27 November 2014; revised 26 December 2014; accepted 5 January 2015

Academic Editor: Atsuko Watanabe, Saitama Medical University, Japan

Copyright (C) 2015 by authors and Scientific Research Publishing Inc.

This work is licensed under the Creative Commons Attribution International License (CC BY). http://creativecommons.org/licenses/by/4.0/

c) (7) Open Access

\begin{abstract}
We report a case of a 7-year-old child who received a peripherally inserted central catheter (PICC) for chemotherapy. He suffered from an unusual PICC fracture in vivo with pulmonary embolism after eight circles of chemotherapeutic agents administered. In this case report, we review the literatures for the underlying pathophysiology of this uncommon phenomenon. IV nurses should consider the possibility of the infusion dysfunction deriving from the PICC line fractured. Accurate tip catheter placement and specific care may reduce morbidity.
\end{abstract}

\section{Keywords}

Peripherally Inserted Central Catheter, Fracture, Children, Complication

\section{Introduction}

Peripherally inserted central catheters (PICC) are being used with increasing frequency in the pediatric population. It provides an important long-term vascular access for the safe administration of chemotherapeutic agents, or those irritating drugs with a high osmolality or a nonphysiological $\mathrm{pH}$ damaging to peripheral vessels. However, despite that PICCs have been shown to have the lower risk of complications when compared with tunneled central venous lines, it has been associated with a number of device-specific complications that manifest during insertion while the line is indwelling. PICC fracture has been reported in a few cases caused by its silicone ma- 
terial, duration of the catheter and the quality of care of these lines, including the pediatric population [1]. To our knowledge, almost catheters were reported to be fractured in vitro at or near the entrance site. In this report, we describe one case of an unusual PICC fractured in vivo with embolization happening in a child.

\section{Case Report}

A 7-year-old boy diagnosed with acute lymphocytic leukemia had a peripherally inserted central catheter (PICC) line procedured for chemotherapy. The patient was recorded normal nutrition status with $26 \mathrm{~kg}$ weight and 118 $\mathrm{cm}$ height. A single-lumen 18-guage (4 Fr) catheter (Bard Groshong, USA) was inserted via the basilic vein of the left arm at the point of $0.5 \mathrm{~cm}$ below elbow crease on December 22, 2010. Postoperative routing chest radiograph showed the tip position of the line was at the beginning of the superior vena cava (SVC) with $37 \mathrm{~cm}$ long inserted (Figure 1). Blood withdraw and infusion though the catheter all did well. Before discharging from the first therapy scheme with the PICC line in situ, the patient's parents were received the education of the catheter maintenance skills. Because of the far distance from their residence to the specialized clinic, the line routine flushing and re-steriled dressing care had to be given by family members or local non-specialized nurses, further simplifying outpatient treatment such as antibiotic infusions. During successful eight plans of chemotherapy administated, the patient suffered from febrile seizures during neutropenia period, while his parents complained the difficulty of the tube flushing. When the patient attended the pediatric chemotherapy unit to receive the next cycle of treatment on November 15th, 2011, the charge nurse found the catheter be difficultly aspirated and flushed, infusion drop rate via the line decreased obviously than before. The patient had not noticed any leakage or other changes at the insertion site, and had no respiratory distress or arrhythmia disorders. Occlusion is the most likelihood complication practically happened in the catheter. Condsidering of the nearly one-year dwell time and the possibility of catheter migration or thrombosis, chest X-ray films were taken before urokinase medication, which showed that the catheter was fractured inside at axillary section. The fluoroscopy, angiograms immediately were taken to get the exact position of the embolized line fragment (Figure 2). It revealed the fractured piece of PICC line lying in the left main pulmonary artery (arrows 1 and 2) with both ends in the upper and basal segment branches in the left lobe, while the point of fractured line distal fragment (arrow 3) remained in the axillary venous. Fortunately, the fractured fragment embolism was successfully snared by percutaneously inserted femoral catheter, the remained line was removed intactly. No further complications appear after catheter removal. On checking the embolized fragment of the fractured catheter, we found there has been a $1 \mathrm{~cm}$ long blood thrombosis at the point of fractured fragment inside (arrow 1).

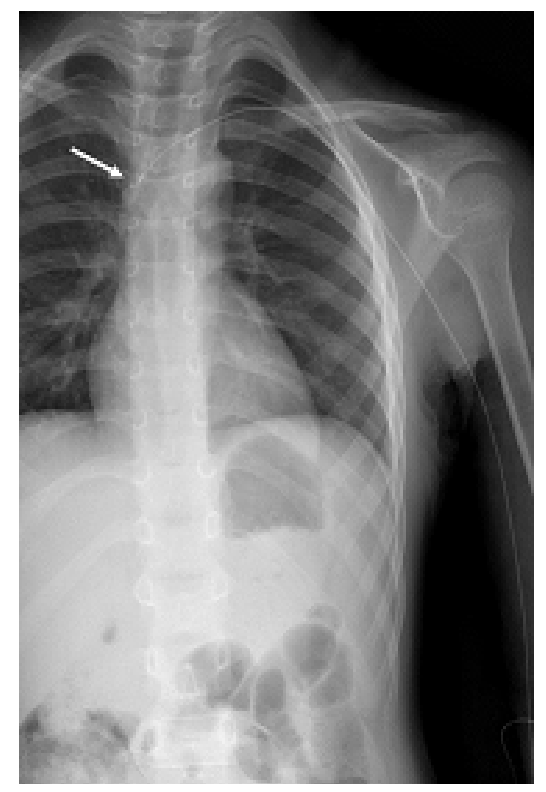

Figure 1. The tip of the PICC was located at the beginning of the superior vena cava (SVC) after inserting. 


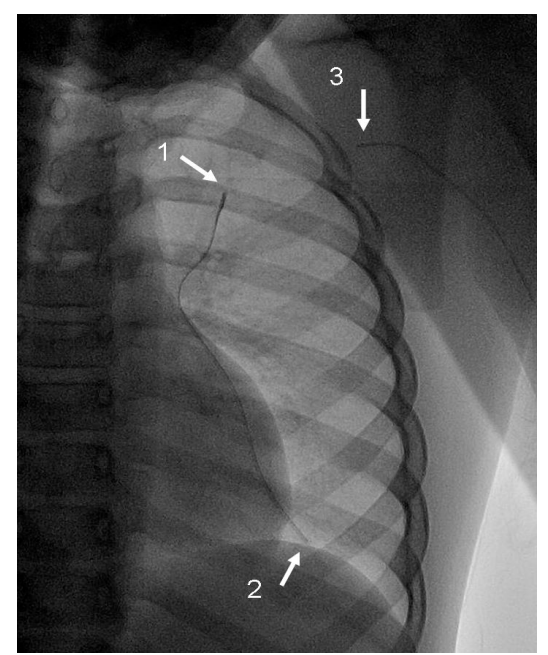

Figure 2. The chest angiograms immediately taken after X-ray films. Fractured piece of PICC line in the left main pulmonary artery is noted. Both ends (arrows 1 and 2) of the fractured fragment are in the upper and basal segment branches in the left lobe, while the remained line fractured distal fragment (arrow 3 ) is located in the axillary venous.

\section{Discussion}

PICCs have become a popular vascular access device in children requiring long-term IV access. Postinsertion complications were recorded including breakage/leakage, phlebitis, catheter-related infection (CR-I), thrombosis, and accidental removal [2]. Fracture and embolization are very rare happened comparing with other complications, but can cause potentially serious clinical disorders such as pulmonary embolism, cardiac arrythmias, cardiac perforation and sepsis [3]. Almost cases of catheter fracture were reported at the entrance site just distal to the cuff at the point, where the caliber of the line narrows [4]. We report this unusual case of PICC fracture in vivo with embolization accidently happened in pediatric patient without symptoms. It's easily confused to consider the complication of occlusion happened for simply presenting with decreased infusion rate and difficulty withdrawing from the line.

The tip of PICC should be put in the lower third of the superior vena cava (SVC) proximal to the right atrium. A "short" catheter was called for the catheter whose tip is located in the upper or middle third of the SVC or in the innominate veins [5], as in this case. Formation of a fibrin sleeve around the short catheter occurs more frequently with persistent withdrawal occlusion and ball valve obstruction [6]. The $1 \mathrm{~cm}$ blood embolism found inside the point of the fractured fragment showed the thrombotic occlusion happened before long. In fact, occlusion evidence was given by the parents for difficulty flushing and withdrawing from the catheter during outpatient period. Thrombosis happened in this case may be easily explained by changed intrathoracic pressure from febrile seizures history occasionally. For the patient discharged home with the catheter in situ cared by his family, the dysfunctional catheter was likely power-injected for attempts to flush the obstruction by non-special persons. These sillicone catheters such as the 3-way valve Bard Groshong PICC were shown to tolerate power injection pressure between 95 - 125 psi, inadequate power injection of PICCs might result in catheter rupture [7]. In additional, the likelihood of any catheter-related complication may occur with time for catheter fatigue [4]. The catheter in this case stayed for 328 days, remarkly more than the average dwelling time of 246 days which is longer than any previously reported studies for children [2]. The exact explaination of this case is not well understood and many hypotheses have been strongly proposed of the partial obstructive catheter ruptured by force procedure.

The malposition of PICC catheter may be explained by inaccurate anthropometric measurements. These measurements estimate the desired length of the catheter in association with the site of venipuncture, the venous choice and the height of the patient. Anthropometric method is quite precise in estimating the length of the catheter in the adult patients, but not in children. PICC catheters should be put more suitably in right arm for accurate tip location. If the fluoroscopy arranged, PICC nurses may perform the catheter insertions in the im- 
age-guided therapy unit, available for early check of the tip position.

\section{Conclusion}

In conclusion, health care professionals need to be more aware of this phenomenon and signs to observe for: difficult flushing or blood aspiration from the PICC line, infusion gradually dysfunction. An expert PICC nurse team should take charge with catheter dressing care for outpatients to minimize the incidence of PICC-related complications. Prompt investigations like chest roentgenogram including a view of the arm with the insertion site should be taken to check the integrity and position of the PICC line. Furthermore, fluoroscopy use may be suitable for easily checking the line in children by reducing the radiation exposure.

\section{References}

[1] Kossoff, E.H. and Poirier, M.P. (1998) Peripherally Inserted Central Venous Catheter Fracture and Embolization to the Lung. Pediatric Emergency Care, 14, 403-405. http://dx.doi.org/10.1097/00006565-199812000-00006

[2] Shen, G.M. and Gao, Y.J. (2009) Survey of the Long-Term Use of Peripherally Inserted Central Venous Catheters in Children with Cancer Experience in a Developing Country. Journal of Pediatric Hematology/Oncology, 31, 489-492. http://dx.doi.org/10.1097/MPH.0b013e318190d740

[3] Tan, P.L. and Gibson, M. (2006) Central Venous Catheters: The Role of Radiology. Clinical Radiology, 61, 13-22. http://dx.doi.org/10.1016/j.crad.2005.07.010

[4] Chow, L.M., Friedman, J.N., Macarthur, C., Restrepo, R., Temple, M., Chait, P.G. and Connolly, B. (2003) Peripherally Inserted Central Catheter (PICC) Fracture and Embolization in the Pediatric Population. Journal of Pediatrics, 142, 141-144. http://dx.doi.org/10.1067/mpd.2003.67

[5] Caers, J., Fontaine, C., Vinh-Hung, V., De Mey, J., Ponnet, G., Oost, C., Lamote, J., De Greve, J., Van Camp, B. and Lacor, P. (2005) Catheter Tip Position as a Risk Factor for Thrombosis Associated with the Use of Subcutaneous Infusion Ports. Support Care Cancer, 13, 325-331. http://dx.doi.org/10.1007/s00520-004-0723-1

[6] Pittiruti, M., Scoppettuolo, G., La Greca, A., Emoli, A. and Brutti, A. (2008) The EKG Method for Positioning the Tip of PICCs: Results from Two Preliminary Studies. JAVA, 13, 4.

[7] Coyle, D. and Bloomgarden, D. (2004) Power Injection of Contrast Media via Peripherally Inserted Central Catheters for CT. Journal of Vascular and Interventional Radiology, 15, 809-814. http://dx.doi.org/10.1097/01.RVI.0000128812.20864.EC 
Scientific Research Publishing (SCIRP) is one of the largest Open Access journal publishers. It is currently publishing more than 200 open access, online, peer-reviewed journals covering a wide range of academic disciplines. SCIRP serves the worldwide academic communities and contributes to the progress and application of science with its publication.

Other selected journals from SCIRP are listed as below. Submit your manuscript to us via either submit@scirp.org or Online Submission Portal.
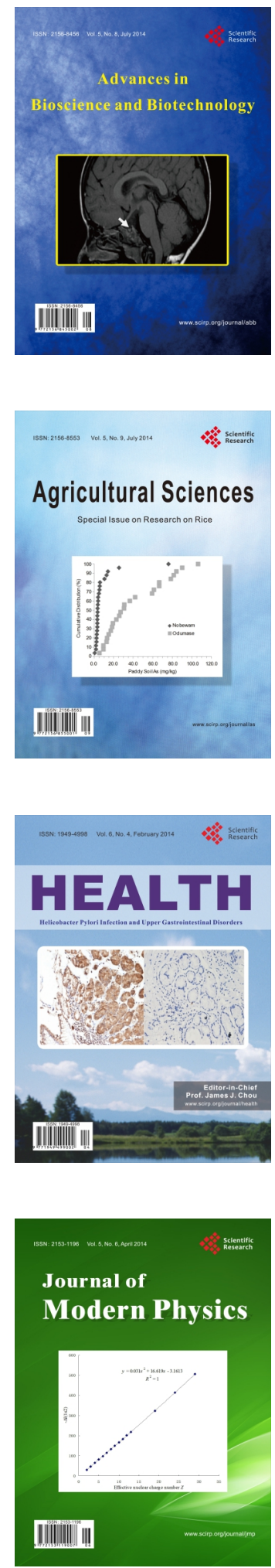
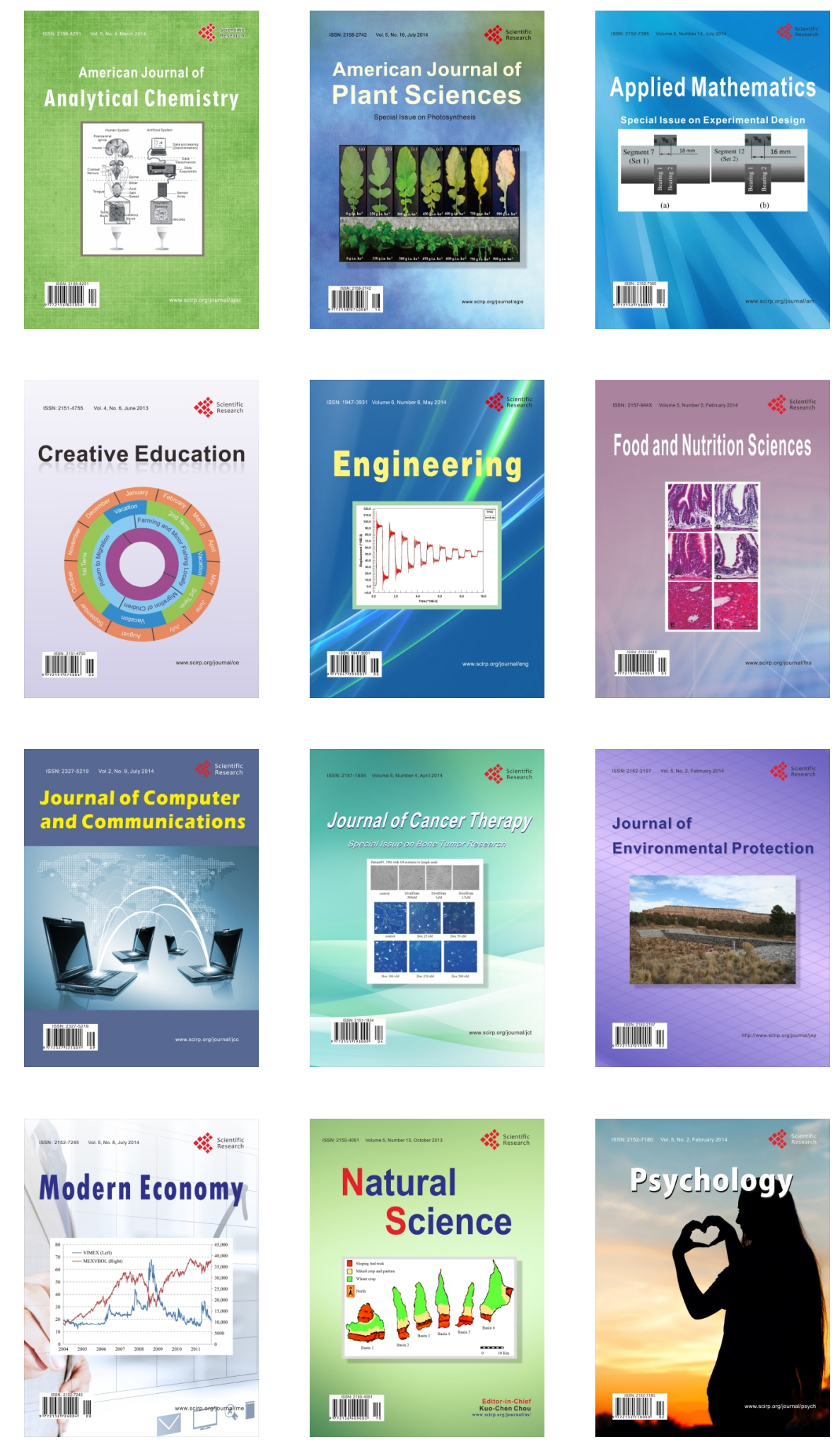